\title{
Evaluation of radiative power loading on WEST metallic in-vessel components
}

\author{
M-H. Aumeunier ${ }^{1}$, P. Moreau, J. Bucalossi, M. Firdaouss \\ CEA/IRFM \\ F-13108 Saint-Paul-Lez-Durance, France \\ E-mail: marie-helene. aumeunierlcea.fr
}

In nuclear fusion devices, the in-vessel components have to withstand high heat loads coming both from conducted particles and from plasma radiations. This paper aims at evaluating the radiative power loading in the new fully metallic and highly reflective environment of WEST (Tungsten (W) Environment in Steady State Tokamak). Indeed, because of multiple reflections, some critical elements, which are not directly exposed plasma radiative flux, can be impacted by photon irradiation and receive significant radiative heat power. Predictive calculations based on Monte Carlo ray tracing photonic simulation in a representative $3 \mathrm{D}$ geometry have been carried out to check the heat loads at any location in the vacuum vessel, and this by considering different plasma scenarios. The results have been used to validate and better define the design of the WEST in-vessel components by identifying critical elements requiring additional heat flux protection such as the vacuum vessel or the baffle supporting structure.

First EPs Conference on Plasma Diagnostics - $1^{\text {st }}$ ECPD

14-17 April 2015,

Villa Mondragone, Frascati (Rome) Italy

\footnotetext{
${ }^{1}$ Speaker
} 


\section{Introduction}

For actively cooled machine aiming at long pulse operation such as WEST and ITER, operational limits are fixed by the heat power load on plasma facing components (PFCs). The heat loads come mostly from conducted particles along magnetic field lines which causes power flux deposits up to $20 \mathrm{MW} / \mathrm{m}^{2}$. This paper investigates a second source coming from the plasma radiations and aims at evaluating their impact on metallic materials behavior. So far, for the fusion devices using carbon materials with high emissivity $(\varepsilon>0.8)$, it was usually assumed that all the radiative power flux emitted by the plasma is absorbed by the PFCs. With the recent use of highly reflective $(\varepsilon \sim 0.3)$ metallic components, the evaluation of the radiative power deposition is made much more complex due to the multiple reflections. In particular, this can affect unexpectedly some critical elements, which are not directly exposed plasma radiative flux.

The WEST (Tungsten (W) Environment in Steady State Tokamak) project is a major upgrade of Tore Supra tokamak to transform it into an X-point magnetic configuration machine with a fully metallic environment, aiming at testing ITER technologies in relevant plasma conditions [1]. This paper aims at evaluating the radiative power density on components which are not directly exposed to plasma radiation and hence not designed for high heat flux and to provide a more accurate heat flux evaluation on exposed PFCs as well. Getting a quantitative prediction of flux deposit, requires a sophisticated approach taking into account the 3D geometry of full tokamak and a model of photon-materials interactions.

\section{Modelling principle}

The photonic simulation code is based on a Monte Carlo ray tracing (SPEOS CAA V5 Based from OPTIS Co., http://www.optis-world.com ) able to propagate the ray through the complex geometry of tokamak and taking into account the multiple inter-reflections of the ray in the vacuum vessel. The process uses forward simulation which consists in casting the rays directly from source and tracing their paths (with reflections) to the 3D objects. The power flux originating from sources and falling on geometry is provided in irradiance $\left(\mathrm{W} / \mathrm{m}^{2}\right)$, varying with respect to the cosine of the incident angle. The results are displayed as a 3D map from which we can extract the irradiance/absorption value for each mesh node. The total absorbed flux (in Watt) is computed after surface reconstruction from this set of nodes.

\subsection{Modelling of radiative sources}

During the plasma operation, two kinds of radiative power source can be considered: the plasma radiations with a power rating of several megawatts; the thermal radiations of PFCs which exhibit high temperature due to interaction with conducted particles.

The plasma radiations are considered to originate both from: the core plasma and the divertor region. They have been modelled as two torus respectively of minor radius $20 \mathrm{~cm}$ and $10 \mathrm{~cm}$ and located close to the core plasma center $(\mathrm{R}=2.5 \mathrm{~m} ; \mathrm{Z}=0 \mathrm{~m})$ and the $\mathrm{X}$ point position ( $\mathrm{R}=2.25 \mathrm{~m} ; \mathrm{Z}=-0.65 \mathrm{~m})$. The plasma is considered as a transparent medium (without absorption) and is assumed to be an isotropic source emitting mostly in the ultra-violet region of the spectrum. 
The secondary thermal sources are localized mainly in the divertor in contact with the plasma through the strike points. We reproduce the far X-point plasma configuration when the heat pattern on lower divertor can be modelled as two toroidal $12 \mathrm{~mm}$ wide strips separated of $20 \mathrm{~cm}$ with a temperature peak of $2000^{\circ} \mathrm{C}$. Even in this case of high heat flux, the contribution of thermal radiations coming from PFCs remains insignificant with a total flux power evaluated lower than $10 \mathrm{~kW}$. Thus the rest of paper focuses on the primary sources coming from plasma radiations.

\subsection{WEST tokamak geometry}

In the study reported here, the 3D geometry of WEST is modelled with the following elements (Figure 1):

- vacuum vessel modelled as a $360^{\circ}$ revolution solid $(\mathrm{R}=2.4 \mathrm{~m} ; \mathrm{a}=0.72 \mathrm{~m})$,

- lower and upper divertor modelled as plates tilted at $22^{\circ}$. The lower divertor receives the heat flux conducted by the plasma.

- the baffle and its supporting structure,

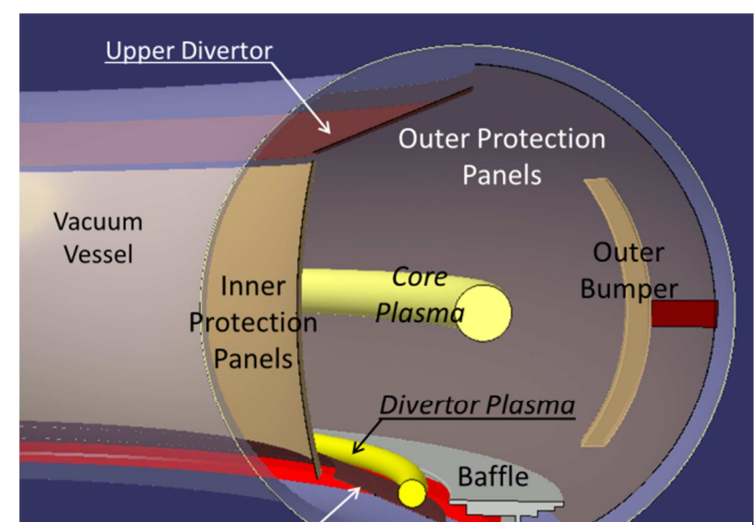

Figure 1: Simplified 3D geometry of WEST tokamak - inner and outer protection panels in stainless steel,

- outboard bumper modelled in a local sector of machine, since it is also a critical component close to plasma with a large front surface (of $35 \mathrm{~cm}$ wide and $1 \mathrm{~m}$ height).

\subsection{Reflectivity issue}

A main challenge of this work is the modelling of material optical properties which define how light interacts with geometry. We assume that all in-vessel components are completely opaque to the plasma radiated power. The light behaviour incident at their surface is fully described by the Bidirectional Reflectivity Distribution Function (BRDF). The BRDF gives the reflectance of a surface with regards to the wavelength, the angle of incident of incoming light and of the output light. The reflection model used in this paper results of a combination of three main components: 1) lambertian component: the probability to be reflected in a given direction is the same for all the direction of the space, 2) specular component: the proportion of reflected flux is given by the Fresnels law; 3) gaussian component: the reflected light has a Gaussian probability to be reflected with a particular angle around the main direction defined by Snell Descartes laws. Simulations have been performed considering highly specular model (2\% lambertian) following a Gaussian shape with a full-width half maximum of $12^{\circ}$, roughly adjusted to the experimental data in [2]. The BRDF is assumed to be the same over all the spectral range and for any illumination geometry. However, such models should be considered with caution since it remains difficult to assess the BRDF and the material reflectance in real conditions. Thus the results of Section 3 consider two limiting cases of reflectivity when the total hemispherical reflectivity $\mathrm{R}$ varies between $\mathrm{R}=0(\mathrm{e}=1)$ and $\mathrm{R}=0.5(\mathrm{e}=0.5)$ for all in-vessel components surface. 


\section{Results}

\subsection{Validation of simulation}

As a first step, validation of the SPEOS calculation has been carried out. It consisted in checking the conservation of the radiated power density by summing the power flux falling on all in-vessel components. First simulations considering a $10 \mathrm{MW}$ power radiative source allowed checking that the total input power is recovered within $0.1 \%$ error considering absorbing materials $(\mathrm{R}=0)$ and within $1 \%$ error considering reflective materials $(\mathrm{R}=0.5)$. A second step has been performed to get an adapted mesh size and the rays number $N$ ensuring to optimize computations time. A good compromise between accuracy and computations time is found with 200 million rays number and a mesh cell size about of $60 \mathrm{~mm}(0.5$ million of mesh nodes).

\subsection{Simulation of WEST reference scenario}

Regarding the magnetic configuration, the plasma volume emitting the higher radiations can be either localized in the chamber center in mid-plane or in the divertor region near to the strike X-point- as shown in Figure 1. Typical WEST plasma scenarios will be a combination of these two radiated source with a total injected power of $10 \mathrm{MW}$. Figure 2 shows the incident radiative power flux on different part of the in-vessel machine in two limiting cases $\mathrm{R}=0$ and $\mathrm{R}=0.5$ for typical WEST scenario (6MW core plasma, $4 \mathrm{MW}$ divertor plasma). On one hand, reflections within metallic machine have the advantage to redistribute more uniformly the plasma radiations, leading to a reduction of the radiative power load by a factor up to $2 / 3$ on lower divertor very close to plasma source. On the other hand, this causes also excessive power flux density on components which are not directly exposed to plasma radiations. This may be especially critical for uncooled components such as the baffle support, the vacuum vessel and the back face of outer bumper where are located a few diagnostics not designed for high heat flux. In the rest of paper, the contribution of the plasma and divertor radiative is evaluated separately. The flux coming onto a component is simply the sum of these 2 contributions plus the IR radiations of PFCs.
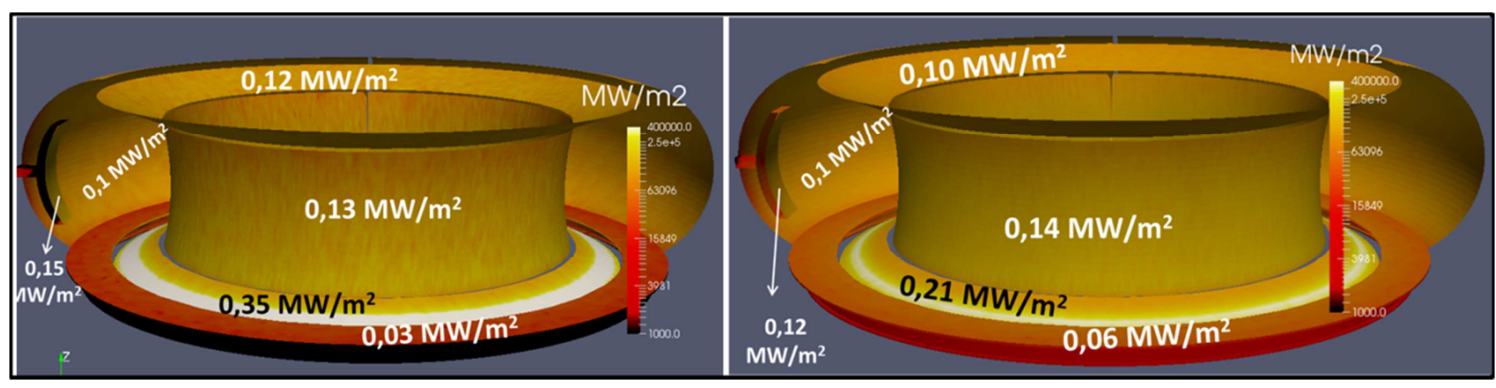

Figure 2: Radiative power loading (6MW core $+4 M W$ divertor plasma). Left: $R=0$ Right $: R \sim 0,5$

Figure 3 summarizes the predicted mean radiative power on components characterized by a uniform absorption distribution over the surface. The upper divertor is designed to withstand high heat loads (a few $\mathrm{MW} / \mathrm{m}^{2}$ ) induced by the conducted particles, which makes negligible the contribution of plasma radiation (lower than $0.2 \mathrm{MW} / \mathrm{m}^{2}$ ). On the inner and outer protection panels, the heat fluxes with $\mathrm{R}=0$ and $\mathrm{R}=0.5$ are similar, of the order of $140 \mathrm{~kW} / \mathrm{m}^{2}$, which is 
quite higher than the conducted power load (these components have been designed to sustain such heat flux). Nevertheless, this value is significant for plasma facing mirror (170 $\mathrm{mm}$ diameter) of the infrared thermography system, located in an opening in the internal protection panel, which lead to design an adequate system to extract the heat load [3]. Last, the maximum radiative power flux is estimated at $40 \mathrm{~kW} / \mathrm{m}^{2}$ for the back face of outer limiter and the diagnostics will have

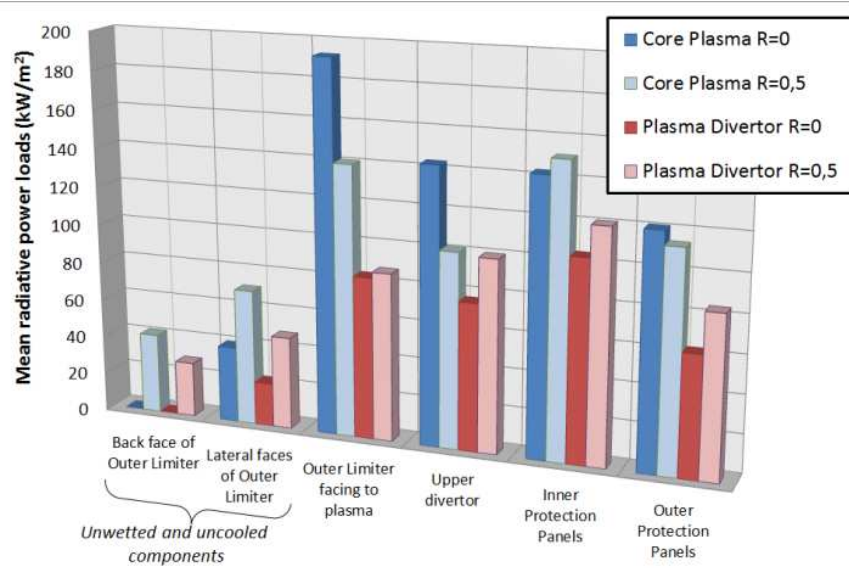

Figure 3: Mean radiative power load for 10MW divertor plasma (red) and core plasma (blue) to be designed accordingly.

Figure 4 and Figure 5 show the non-uniform radiative power flux profile respectively along the vacuum vessel and baffle support which are not design to sustain heat flux. The most strongly affected region by the reflected photonic flux coming from plasma radiation is the high field side of baffle support, for which the radiative heat load can reach up to $100 \mathrm{~kW} / \mathrm{m}^{2}$ on the line segment $[\mathrm{EF}]$ (Fig. 5). Likewise, because of the gap between the lower divertor and the baffle, the vacuum vessel is quite exposed to plasma radiations with a peak of heat flux at $100 \mathrm{~kW} / \mathrm{m}^{2}$ below the baffle (Fig.4). These predictive results led to integrate actively cooled extra protection panels for the baffle support and the vacuum vessel as illustrated in Fig.1 (red surface below the baffle). This allowed reducing significantly the radiative heat power density on the line segment $[\mathrm{EF}]$ of baffle support to $20 \mathrm{~kW} / \mathrm{m}^{2}$ and the peak value on vacuum vessel below the baffle by a factor two.

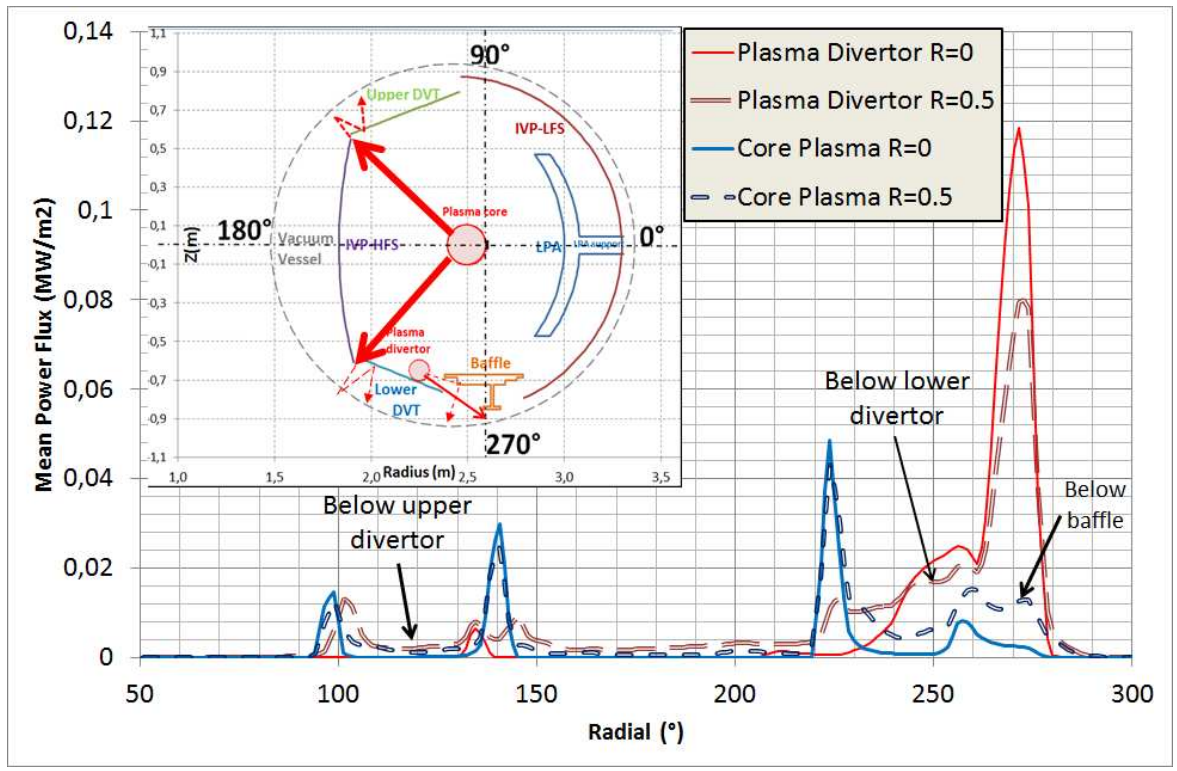

Figure 4: Comparison of the poloidal radiative flux profile of vacuum vessel for $R=0$ (solid line) and $R=0,5$ (dotted lines) for divertor plasma (red curves) and core plasma (blue curves) 


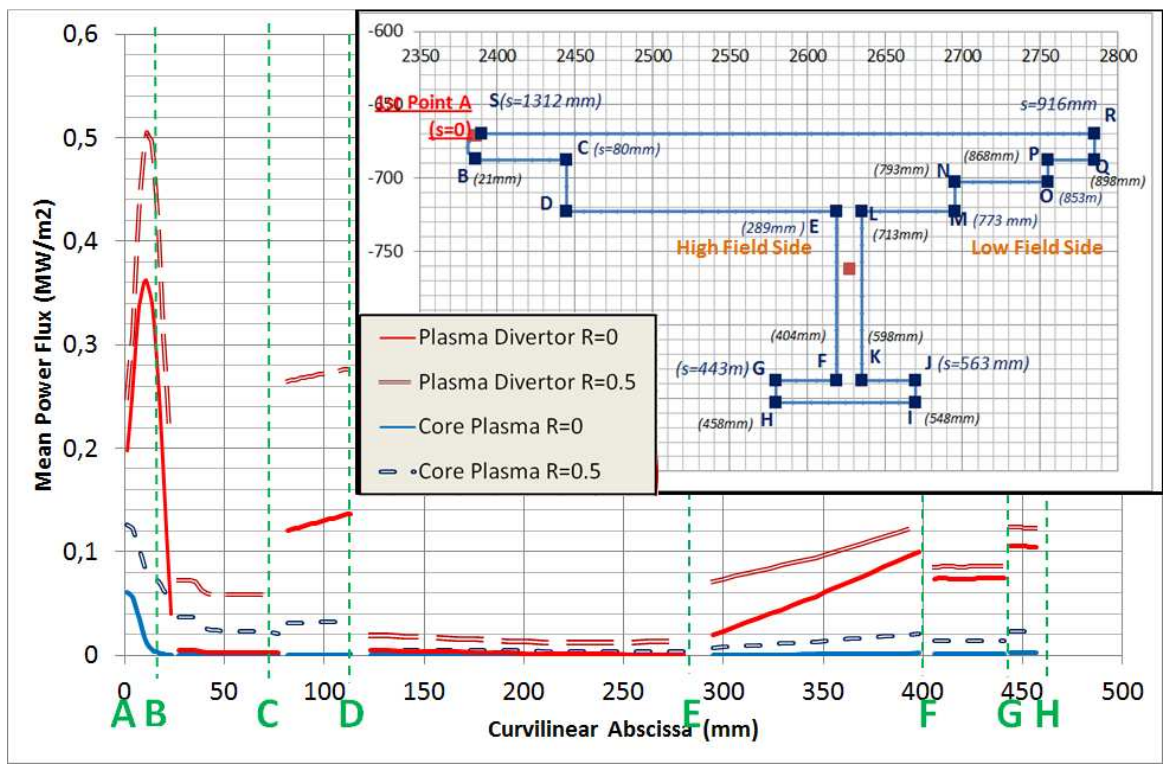

Figure 5: Comparison of the poloidal radiative flux profile of baffle support for $R=0$ (solid line) and $R=0,5$ (dotted lines) for divertor plasma (red curves) and core plasma (blue curves)

\section{Conclusion}

A detailed study of the radiative power loading has been thus performed on critical components of WEST which are not designed for high heat flux but which are potentially exposed due to multiple reflections. This study is based on 3D Monte Carlo ray tracing code and first verification has been carried out proving the capability of this approach. This study has allowed validating the design of in-vessel component in terms of heat flux extraction capability and has proposed additional protection for some uncooled elements. This study reported in this paper was mainly motivated for engineering design purposes but photonic simulation is also applied for diagnostics measurement issues in order to better understand the contribution of reflected flux that interferes with useful signal [4]. Indeed, false interpretation of diagnostics due to the presence of reflections can limit the operation of machine (by triggering false alarms) or endanger the in-vessel components (by not detecting hot spot). Based on this photonic simulation, the implementation of synthetic diagnostic including optical response is on-going and will be used for improving the understanding of WEST wall monitoring systems.

\section{References}

[1] J. Bucalossi et al., Feasibility study of an actively cooled tungsten divertor in Tore Supra for ITER technology testing, Fusion Engineering and Design 86, 684-688 (2011)

[2] R. Reichle, et al., Infrared reflection properties and modelling of in situ reflection measurements on plasma-facing materials in Tore Supra, Phys. Script., vol. T138, p. 014029 (2009)

[3] F. Micolon et al., Mechanical design and thermo-hydraulic simulation of the infrared thermography diagnostic of the WEST tokamak, Fusion Engineering and Design, ISSN 0920-379, 2015

[4] M-H. Aumeunier et al., Modeling of the ITER-like wide-angle infrared thermography view of JET, Rev. Sci. Instruments 83, 10D522 (2012) 